\title{
Erratum: Classical Approach to Multichromophoric Resonance Energy Transfer [Phys. Rev. Lett. 115, 110402 (2015)]
}

Sebastián Duque, Paul Brumer, and Leonardo A. Pachón

(Received 19 May 2016; published 9 June 2016)

DOI: 10.1103/PhysRevLett.116.239903

We are grateful to Mr. Simon Axelrod and Dr. Aurelia Chenu for identifying a numerical error in the computer program producing Fig. 2 of original Letter. Correcting this error yielded improved results, as shown here in the figure. Specifically, fitting the data with the functional form $\left(a e^{-k_{f} t}+b e^{-k_{u f} t}+c\right) /(a+b+c)$ used in the text yielded constants $a=b=1$, $c=2.01$, a fast decay component of $k_{u f}=13.52 \mathrm{ps}^{-1}$, and an electronic energy transfer rate of $k_{f}=1.11 \mathrm{ps}^{-1}$. The latter, compared to the value of $0.92 \mathrm{ps}^{-1}$ reported in the text, is seen to be in even better agreement with the experimental value of $1.25 \mathrm{ps}^{-1}$.

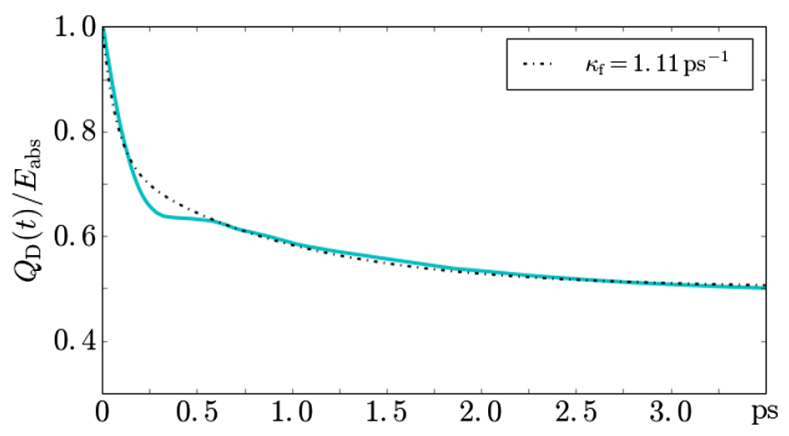

FIG. 2. Normalized total energy within the B800 ring (donor) in LH2 initially excited with a delta pulse. Dashed curve is a best fit to the oscillatory (cyan) result. 